\title{
Potential Role of Monocyte Chemoattractant Protein-1 in Monitoring Disease Progression and Response to Treatment in Overactive Bladder Patients
}

\author{
Gamal Ghoniem ${ }^{1}$, Bilal Farhan ${ }^{1,2}$, David Csuka ${ }^{1}$, Frank Zaldivar ${ }^{3}$ \\ ${ }^{1}$ Department of Urology, University of California, Irvine (UCI), Orange, CA, USA \\ ${ }^{2}$ Urology Division, Medical Branch (UTMB), University of Texas, Galveston, TX, USA \\ ${ }^{3}$ UCI Institute for Clinical \& Translational Science (ICTS), Irvine, CA, USA
}

Purpose: To compare urinary levels of monocyte chemoattractant protein-1 (MCP-1), an inflammatory cytokine, in healthy controls and overactive bladder $(\mathrm{OAB})$ patients, to correlate changes in urinary $\mathrm{MCP}-1$ with $\mathrm{OAB}$ treatment response and symptom severity, and to study the diagnostic potential of MCP-1 for OAB, as well as the efficacy of MCP-1 as a potential biomarker for different phenotypes of OAB.

Methods: We used enzyme-linked immunosorbent assay to measure normalized urinary MCP-1 levels in 56 individuals (43 $\mathrm{OAB}$ patients and 13 controls). We assessed the $\mathrm{OAB}$ patients at 3 visits with 2 validated symptom severity questionnaires (OAB-V8 and Patient Perception of Bladder Condition).

Results: The mean pretreatment urinary MCP-1 level at visit 1 (229.2-pg/mg creatinine) was significantly greater than the MCP-1 levels at visit 3 in both the treatment $(107.0-\mathrm{pg} / \mathrm{mg}$ creatinine) $(\mathrm{P}<0.001)$ and control $(52.35-\mathrm{pg} / \mathrm{mg}$ creatinine) groups $(\mathrm{P}<0.001)$. Average $\mathrm{OAB}$ symptom severity decreased significantly from visit 1 (baseline) to visits 2 (4 weeks) and 3 (12-14 weeks) and was significantly correlated with urinary MCP-1 levels. Urinary MCP-1 levels dropped significantly $(\mathrm{P}=0.002)$ posttreatment in patients whose symptom severity improved by $>30 \%$, whereas nonresponders displayed no significant MCP-1 decrease $(\mathrm{P}=0.164)$. The receiver operating characteristic analysis of the $\mathrm{OAB}$ visit 1 and control groups produced an area under the curve of 0.891 . We found no significant differences in sex, race, or age between the OAB and control groups. Conclusions: MCP-1 levels differed significantly between the control and OAB groups and were closely correlated with symptom severity and treatment response. The good diagnostic accuracy of MCP-1 for OAB suggests the potential usage of MCP-1 for $\mathrm{OAB}$ diagnosis. The varying response of urinary MCP-1 levels to treatment may indicate at least 2 potential phenotypes of OAB. MCP-1, in combination with other biomarkers and symptom severity questionnaires, could potentially aid in developing a patient-centered $\mathrm{OAB}$ treatment approach.

Keywords: Chemokine CCL2; Urinary bladder, Overactive; Cytokines

- Fund/Grant Support: The data collection and equipment costs of this project were in part supported by the National Center for Research Resources and the National Center for Advancing Translational Sciences, National Institutes of Health, through Grant UL1 TR001414.

- Research Ethics: This study was approved by the Institutional Review Board of University of California Irvine (approval number: 2014-1355).

- Conflict of Interest: Gamal Ghoniem: Research grant (Cogentix/Laborie), Consulting: RebeccaTech and Boston Scientific. Bilal Farhan, David

Csuka, Frank Zaldivar: have no conflicts of interest to disclose.

Corresponding author: Gamal Ghoniem (iD https://orcid.org/0000-0002-9028-7783 Department of Urology, University of California, Irvine, 333 City Boulevard West, Suite 2100, Orange, CA 92868, USA

E-mail: gghoniem@hs.uci.edu

Submitted: June 11, 2020 / Accepted after revision: November 8, 2020 (i) (5) This is an Open Access article distributed under the terms of the Creative Commons Attribution Non-Commercial License (https://creativecommons.org/licenses/by-nc/4.0/) which permits unrestricted non-commercial use, distribution, and reproduction in any medium, provided the original work is properly cited. 


\begin{abstract}
- HIGHLIGHTS
- Urinary MCP-1 levels correlated to overactive bladder symptoms and showed decreased levels after treatment.

- Data suggest different OAB phenotypes.

- Receiver operating characteristic indicate the usefulness of urinary MCP-1 as a marker; alone or in combination with other markers, for patients with $\mathrm{OAB}$.
\end{abstract}

\section{INTRODUCTION}

Overactive bladder $(\mathrm{OAB})$, as defined by International Continence Society (ICS) guidelines, is a not a disease, but rather a complex syndrome characterized by chronic urinary urgency, with or without urge incontinence, usually in combination with frequency and nocturia [1]. Although the precise molecular etiology of $\mathrm{OAB}$ largely remains unclear, numerous studies have recognized the association of $\mathrm{OAB}$ with inflammation, and therefore increased afferent nerve activity within the lower urinary tract. $\mathrm{A} \delta$ and $\mathrm{C}$ neural fibers of $\mathrm{OAB}$ patients become sensitized and respond abnormally to chemical, thermal, and stretch stimuli, causing urinary urgency and frequency. Additionally, levels of serum C-reactive protein, nerve growth factor, and brain-derived neurotrophic factor are higher in $\mathrm{OAB}$ patients than in control subjects [2-6]. The most recent studies of urinary chemokines in $\mathrm{OAB}$ patients have shown monocyte chemotactic protein-1 (MCP-1) to be the most elevated of all compared to controls, suggesting that the extent of chronic OAB-related inflammation may be evaluated by biomarker analyses. The cytokine MCP-1 was chosen in particular for this study because studies examining numerous cytokines found MCP-1 to be not only the most elevated, but also the most specific to OAB. Urinary MCP-1 levels were even more elevated in $\mathrm{OAB}$ patients than in urinary tract infection patients $[7,8]$.

Chemokines are peptides secreted in response to signals such as proinflammatory cytokines. Their primary function is to regulate cell trafficking. Chemokines can be classified into 4 subfamilies: $\mathrm{CXC}, \mathrm{CC}, \mathrm{CX}_{3} \mathrm{C}$, and C. MCP-1/CCL2, a member of the CC chemokine family, is encoded on chromosome 17 and is a potent chemotactic factor for monocytes, which are known to drive inflammatory diseases $[9,10]$. A wide variety of cells, including endothelial cells, mast cells, and smooth muscle cells, secrete MCP-1 either routinely or after activation by other cytokines [11-13]. In humans, elevated MCP-1 levels have been observed in various inflammatory and autoimmune diseases such as inflammatory bowel disease, multiple sclerosis, diabetes, rheumatoid arthritis, and allergic asthma [14]. Similarly, elevated MCP-1 levels have also been reported in patients with $\mathrm{OAB}$, chronic pelvic pain syndrome, and bladder outlet ob- struction $[7,15,16]$. These findings collectively suggest that abnormal overexpression of MCP-1 contributes to chronic inflammatory conditions in the bladder, such as $\mathrm{OAB}$ and voiding dysfunction. Research into urinary biomarkers may help classify $\mathrm{OAB}$ patients into different phenotypes and assist in understanding the pathophysiological processes that lead to the development of these phenotypes, potentially suggesting targeted therapies based on biomarker levels.

In this follow-up of our original pilot study [17], we used the Quantikine human MCP-1 enzyme-linked immunosorbent assay (ELISA), a validated MCP-1 assay modality for multiple human matrices, to analyze urinary MCP-1 levels in $\mathrm{OAB}$ patients before and after treatment as well as in healthy controls. Our objectives were to confirm the elevation of urinary MCP-1 levels in pretreatment $\mathrm{OAB}$ patients in comparison to posttreatment $\mathrm{OAB}$ patients and controls, to correlate MCP-1 levels with $\mathrm{OAB}$ symptom severity, and to evaluate urinary MCP-1 with respect to the treatment modality and response to treatment. We explored MCP-1 as a potential biomarker for OAB diagnosis in a clinical environment.

\section{MATERIALS AND METHODS}

After obtaining Institutional Review Board approval, both male and female $O A B$ patients $(n=43)$ recruited from a urology specialty clinic and control volunteers $(n=13)$ were enrolled between October 2014 and June 2018. The OAB diagnosis was made based on the clinical ICS definition of OAB, which emphasizes urgency and frequency independently of incontinence [1]. Written consent was obtained from all participants. All patients reporting $\mathrm{OAB}$ symptoms underwent a physical examination and urinalysis.

Patients with other potential causes of OAB symptoms or elevated MCP-1 such as neurogenic bladder, stress urinary incontinence, bladder outlet obstruction, urinary stones, lower genitourinary tract tumors, urinary tract infections, treatable genitourinary conditions, hematuria, postvoid residual volume of $>150 \mathrm{~mL}$, vaginitis, bladder pain syndrome, and renal pathology were excluded from the study. Subjects diagnosed with $\mathrm{OAB}$ before enrollment were required to discontinue $\mathrm{OAB}$ 
medications or neuromodulation for at least 2 weeks before their first visit, following the typical pharmaceutical protocol for treatment washout before a clinical study. The different treatments administered to the $\mathrm{OAB}$ patients included sacral neuromodulation as well as anticholinergics, a beta- 3 agonist (mirabegron), and percutaneous tibial nerve stimulation. All $\mathrm{OAB}$ patients were partitioned into their respective treatment groups based on American Urological Association (AUA)/Society of Urodynamics, Female Pelvic Medicine \&Urogenital Reconstruction (SUFU) treatment guidelines [18]. Controls who had no significant urinary pain (AUA symptom index $<7$ ), no previous diagnosis of $\mathrm{OAB}$, no lower urinary tract symptoms, and no evidence of infection based on negative nitrite dipstick tests were included. While the urine of the control cohort was tested for MCP-1 once, the OAB patients were seen 3 times, at visit 1 (pretreatment baseline), visit 2 (4 weeks after baseline), and visit 3 (12-14 weeks after baseline). For the OAB patients, MCP-1 concentrations were analyzed on visits 1 and 3 . This study was longitudinal only for the $\mathrm{OAB}$ group, as the 1-time control MCP-1 measurement was compared to the OAB group data at both visits 1 and 3 . OAB symptom severity was assessed at all 3 visits with 2 validated questionnaires: the $\mathrm{Pa}$ tient Perception of Bladder Condition (PPBC) questionnaire and the Overactive Bladder (OAB-V8) questionnaire. In both questionnaires, a lower value is indicative of less severe symptoms. Changes in patients' scores on the PPBC and OAB-V8 questionnaires from visit 1 to visit 3 were combined with equal weight into a single average posttreatment percent change to differentiate the $\mathrm{OAB}$ treatment patients into responders and nonresponders. OAB responders displayed a decrease in this composite symptom severity score by more than $30 \%$ from visit 1 to visit 3, while those whose symptoms failed to improve by more than $30 \%$ were considered OAB nonresponders. The $30 \%$ cutoff was a clinical decision, which assumes that a score of 2 out of 7 on a psychometric Likert scale implies meaningful improvement.

After urine collection (midstream, approximately $50 \mathrm{~mL}$ ) from either a control or $\mathrm{OAB}$ patient, the sample was centrifuged at $3,000 \times g$ at $4^{\circ} \mathrm{C}$ for 10 minutes within an hour of collection. Of the resulting supernatant, a $1.5-\mathrm{mL}$ aliquot for MCP-1 testing, as well as a $3.0-\mathrm{mL}$ aliquot for creatinine $(\mathrm{Cr})$ testing were stored at $-80^{\circ} \mathrm{C}$ until analysis. Urinary MCP-1 levels were measured using the Quantikine human CCL2/MCP-1 ELISA kit (R\&D Systems, Cat. \#DCP00, Minneapolis, MN, USA). This is a 3.5- to 4.5-hour solid-phase ELISA test that has been shown to produce accurate MCP-1 concentration results in multiple human sample types. All experimental protocols were performed precisely following the manufacturer's instructions. The average of 2 optical density values for the ELISA-treated urine samples were entered into a 4-parameter calibration curve equation created by performing the experimental protocol on solutions with standard MCP-1 concentrations. The final MCP1 value, as dictated by the calibration curve, was further normalized for metabolic and urine hydration differences via $\mathrm{Cr}$ concentrations. None of the subjects had acute renal pathology or urinary tract infections, so $\mathrm{Cr}$ could be safely used as a representative of metabolic activity; $\mathrm{Cr}$ is often used as a normalization variable in urinary biomarker studies [7]. Therefore, the final unit of MCP-1 was expressed as picograms (pg) of MCP-1/ mg of Cr.

Statistical differences in cohort demographics and mean urinary MCP-1 levels in controls and $\mathrm{OAB}$ patients at visit 1 (pretreatment) and visit 3 (12- to 14 -week posttreatment) were compared using the paired or unpaired 2-tailed t-test for numerical data and the Fisher exact test for categorical data. The paired t-test was always used when the same subject group was compared at visits 1 and 3, whereas the standard t-test was always used when different subject groups were involved. Pearson correlation coefficients and analysis of variance (ANOVA) regression were used to determine the significance of correlations and regression for the relationship between visit $1 \mathrm{MCP}-1$ levels and symptom severity scores. Receiver operating characteristic (ROC) analysis was performed for the visit 1 baseline $\mathrm{OAB}$ patients and control groups to determine the effectiveness of MCP-1 in identifying the presence of OAB. Mean values were calculated and the graph error bars utilized the mean \pm standard error of the mean (SEM). The ROC curve was created using IBM SPSS Statistics ver. 25.0 (IBM Co., Armonk, NY, USA) while all other statistical analyses were performed using Microsoft Excel 2019 (Microsoft Corp., Redmond, WA, USA). P-values $<0.05$ were considered to indicate statistical significance.

\section{RESULTS}

In total, 43 patients with $\mathrm{OAB}$ and 13 controls were enrolled in the study. No significant demographic differences were observed between the $\mathrm{OAB}$ patients and controls in sex, race, or age (Table 1). The mean urinary MCP-1 levels at visit $1(\mathrm{P}=$ $0.593)$ or visit $3(\mathrm{P}=0.219)$ did not differ significantly between 
Table 1. Demographic information

\begin{tabular}{lccc}
\hline Demographic & OAB $(\mathrm{n}=43)$ & Control $(\mathrm{n}=13)$ & P-value \\
\hline Sex & & & 0.730 \\
Male & $11(25.6)$ & $4(30.8)$ & \\
Female & $32(74.4)$ & $9(69.2)$ & 0.161 \\
Race & & & \\
White & $33(76.7)$ & $7(53.8)$ & \\
Other & $10(23.3)$ & $6(46.2)$ & 0.078 \\
Age (yr) & $62.7 \pm 2.53$ & $54.6 \pm 3.62$ & \\
\hline
\end{tabular}

Values are presented as number (\%) or mean \pm standard error of the mean.

P-values for sex and race were computed with the Fisher exact test, whereas the unpaired $t$-test was used for age.

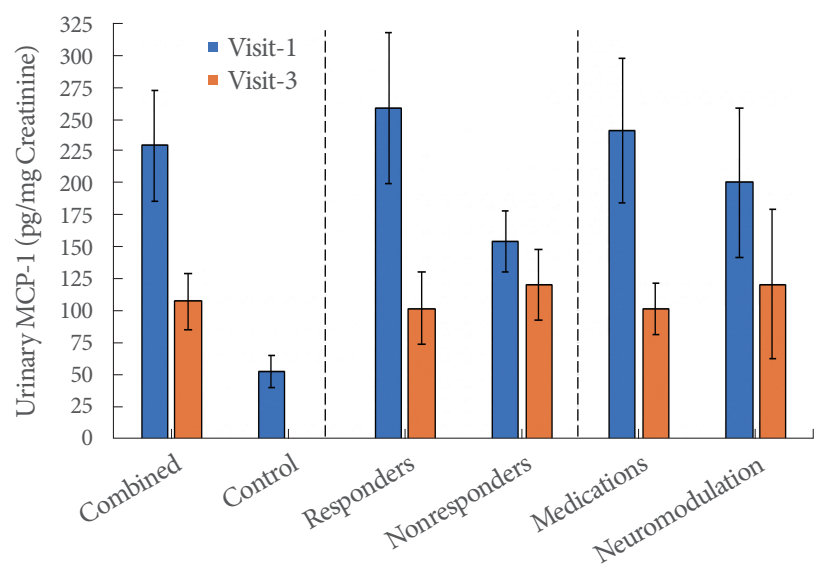

Fig. 1. Monocyte chemoattractant protein-1 (MCP-1) concentration differences for the combined overactive bladder (OAB) treatment, control, responder, nonresponder, medication, and neuromodulation groups for visits 1 and 3. Responders/nonresponders and medications/neuromodulation are different ways of dividing the combined $\mathrm{OAB}$ patient cohort, which includes all subjects besides controls. Combined treatment, all patients received treatment for $\mathrm{OAB}$.

men and women in the $\mathrm{OAB}$ patient cohort. The $\mathrm{OAB}$ treatments consisted of medications (primarily anticholinergics and a beta- 3 agonist) for $72.1 \%$ (31 of 43 ) of the OAB group, and neuromodulation for $27.9 \%$ (12 of 43 ) of the $\mathrm{OAB}$ group.

The average urinary MCP- 1 level of the $\mathrm{OAB}$ patients at visit 1 $(229.2 \pm 43.4 \mathrm{pg} / \mathrm{mg} \mathrm{Cr})$ was significantly higher than both that of the $\mathrm{OAB}$ patients at visit $3(107.0 \pm 21.4 \mathrm{pg} / \mathrm{mg} \mathrm{Cr})(\mathrm{P}<0.001)$ and the control group $(52.4 \pm 13.1 \mathrm{pg} / \mathrm{mg})(\mathrm{P}<0.001)$. The MCP1 level of the $\mathrm{OAB}$ group at visit 3 was also significantly higher than that of the control group $(\mathrm{P}=0.034)$. The $\mathrm{OAB}$ responders $(\mathrm{n}=31)$, as assessed by the symptom severity algorithm dis-

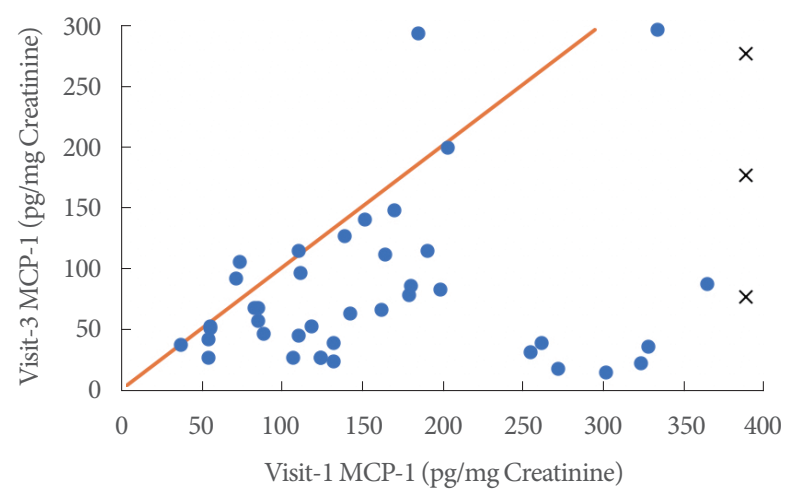

Fig. 2. Urinary monocyte chemoattractant protein-1 (MCP-1) levels at visits 1 and 3 . The diagonal reference line represents identical MCP-1 levels at visits 1 and 3. The 3 crosses represent distant outliers outside of the scatterplot's range; all 3 fell below the extended line nonetheless.

cussed earlier, demonstrated a significant decrease in MCP-1 levels from visit 1 to visit $3(\mathrm{P}=0.002)$, whereas the $\mathrm{OAB}$ nonresponders $(n=12)$ did not demonstrate a significant MCP-1 decrease from visit 1 to visit $3(\mathrm{P}=0.164)$. The MCP-1 levels of the patients who received medication $(\mathrm{n}=31)(\mathrm{P}=0.006)$ and neuromodulation $(\mathrm{n}=12)(\mathrm{P}=0.012)$ both decreased significantly from visit 1 to visit 3 , but this trend was much less pronounced in the neuromodulation group (Fig. 1). Comparisons of visit 1 to visit $1 \mathrm{MCP}-1$ levels $(\mathrm{P}=0.101)$ and visit 3 to visit $3 \mathrm{MCP}-1$ levels $(\mathrm{P}=0.637)$ between responders and nonresponders, as well as for patients receiving medication and neuromodulation ( $\mathrm{P}=0.619$ for visit 1 to visit 1 and $\mathrm{P}=0.765$ for visit 3 to visit 3 ) yielded no significant $\mathrm{P}$-values, implying similar initial and final MCP-1 concentrations between all subdivisions of the total data set.

A comparison of visits 1 and 3 MCP-1 levels against a diagonal line that depicted equivalent MCP-1 levels after treatment produced results that were almost always below the line, indicating that MCP-1 levels predominantly decreased after treatment (Fig. 2).

Additionally, the paired t-test yielded P-values $<0.001$ for all 6 possible comparisons of visit 1 to visit 2 , visit 2 to visit 3 , and visit 1 to visit 3 symptom severity for both the PPBC and OAB$\mathrm{V} 8$ questionnaires, demonstrating steady decreases in symptom severity as the treatment process progressed. Significant ANOVA regression P-values and moderately strong positive correlations were observed between symptom severity and visit 1 MCP-1 levels for both PPBC $(\mathrm{P}=0.005, \mathrm{r}=0.426)$ and OAB-V8 $(\mathrm{P}=0.004, \mathrm{r}=0.436)$ (Fig. 3). 

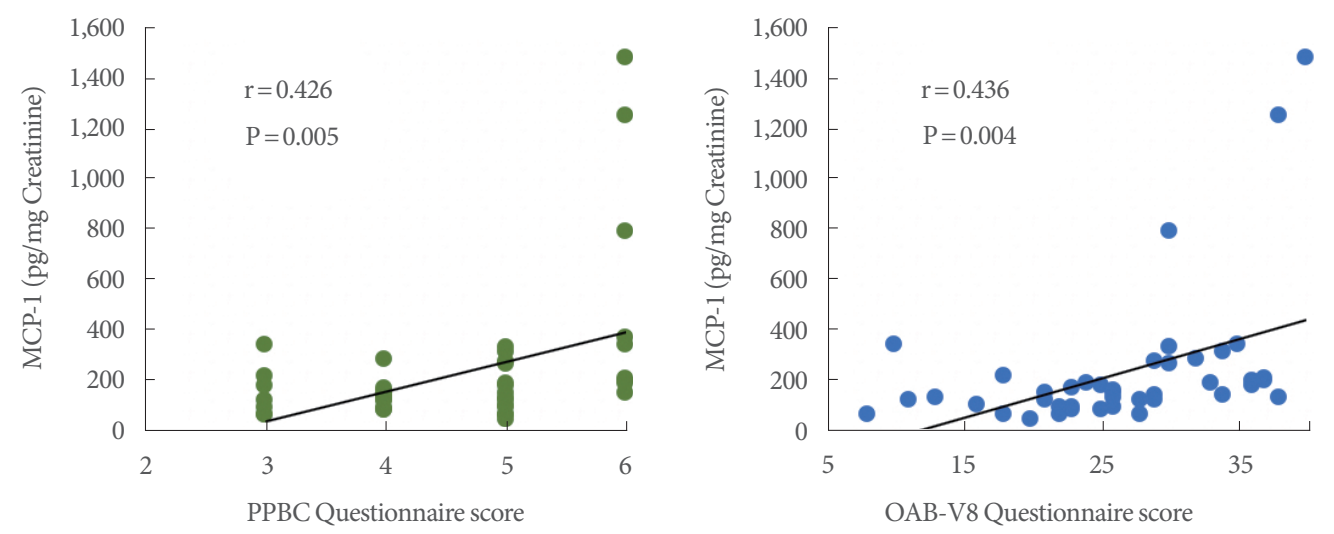

Fig. 3. Relationship between visit 1 monocyte chemoattractant protein-1 (MCP-1) levels and symptom severity questionnaire scores using linear regression, Pearson correlation coefficients, and analysis of variance regression P-values.

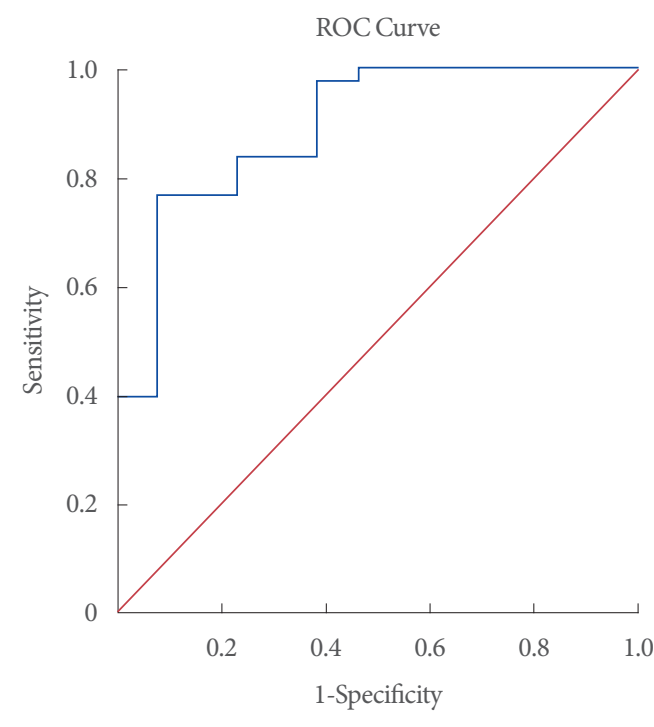

Fig. 4. Receiver operating characteristic (ROC) sensitivity analysis of overactive bladder visit 1 versus control. Area under the curve $=0.891, \mathrm{P}<0.001$.

ROC analysis was performed to measure the diagnostic accuracy of MCP-1 for OAB. (Fig. 4). In conjunction with the area under the curve (AUC), ROC analysis was used to analyze the ability of MCP-1 to differentiate between the pretreatment $\mathrm{OAB}$ patient and control groups.

\section{DISCUSSION}

Extensive research in the medical literature indicates that afferent neuron overactivation triggered by bladder inflammation plays a critical role in the pathogenesis of OAB $[19,20]$. Our study provides strong support for this concept. After 12-14 weeks of treatment at visit 3, OAB patients displayed a significant decrease in mean MCP-1 concentrations when compared to their baseline at visit 1 (Fig. 1). In the vast majority of the $\mathrm{OAB}$ patients, MCP-1 decreased from visit 1 to visit 3 (Fig. 2). Decreases in urinary MCP-1 levels, the inflammatory response, and neural sensitization are all logically associated with successful OAB treatment. Additionally, the average patient symptom severity score decreased significantly from visit 1 to visit 2 to visit 3 for both questionnaires. This result confirms the beneficial effects of OAB treatment for ameliorating patients' symptoms and aligns with the findings of significant correlations between urinary MCP-1 levels and symptom severity for both questionnaires (Fig. 3). Patients with more severe symptoms generally had higher MCP-1 levels, thus suggesting the potential clinical application of using urinary MCP-1 in conjunction with extensive symptom severity assessments to monitor treatment progress. As the pathway between inflammation, neural sensitization, and $\mathrm{OAB}$ symptoms is not entirely understood, it is of considerable importance that an association between the biomarker MCP-1 and OAB symptoms has been established [21]. Because neural sensitization is associated with bladder inflammation, it is possible that since $\mathrm{OAB}$ treatments ameliorate improper neural firing, they also decrease inflammation and MCP-1 levels. Further research is necessary to identify the molecular connections between MCP-1, neural sensitization, and a host of OAB-related mRNAs and miRNAs [22,23] that are integral to the pathogenesis of OAB.

Our data shows that the posttreatment decrease in MCP-1 levels in the symptom responder, medication, and neuromodulation treatment subgroups was significant. In contrast, the symptom nonresponder subgroup did not display a significant 
MCP-1 decrease. The different results between responders and nonresponders add another dimension to the relationship between symptom severity and MCP-1, suggesting that MCP-1 predictably reflects symptom severity not only on the level of a whole sample, but also within particular symptom improvement substrata. MCP-1 could be used as a potential biomarker to evaluate disease progression, as well as response to treatment for OAB. Even though the P-values for both the medication and neuromodulation groups were significant, the neuromodulation group displayed a trend towards a milder posttreatment decrease in inflammation.

After the clinical OAB diagnosis, MCP-1 and other inflammatory analyses could be used to determine the presence of 1 of 2 potential $\mathrm{OAB}$ phenotypes: severe inflammatory $\mathrm{OAB}$, which is associated with high inflammation, intense urgency, urge incontinence, and high MCP-1 levels, or mild inflammatory $\mathrm{OAB}$, which is associated with low inflammation, milder symptoms, and lower MCP-1. The lesser degree to which neuromodulation reduces urinary $\mathrm{MCP}-1$ suggests that medications may be a more effective treatment option for the inflammatory $\mathrm{OAB}$ phenotype, while neuromodulation may be a more suitable for noninflammatory OAB. Currently, there is no targeted therapy available based on the specific pathogenesis, and $\mathrm{OAB}$ diagnosis is based strictly on the symptoms present. However, it will undoubtedly be a promising area for future research to analyze the response of biomarkers to specific therapies and to optimize therapy for these possible OAB inflammatory phenotypes.

The visit 3 MCP-1 level for the OAB group was still significantly higher than in the control group, which means that 1214 weeks of treatment is probably not sufficient to normalize the bladder inflammation associated with OAB completely. These findings highlight the significance of longitudinal $\mathrm{OAB}$ studies using urinary biomarkers and validated symptom questionnaires. A cross-sectional study would only reveal general trends in separate cohorts, not the specific response to treatment over time in any particular patient. Most prior studies of $\mathrm{OAB}$ urinary biomarkers had a small sample size and were cross-sectional. They also failed to account clinical confounders such as race, sex, age, and varying urine hydration levels, and they used equipment designed for the simultaneous processing of multiple cytokines [24]. These factors could explain the finding that MCP-1 levels in OAB patients were found to be over 10 times that of controls [7]. We believe that our results help to narrow down this ratio to just above 4 times that value. This study, which was longitudinal for the OAB cohort and crosssectional for controls, found no significant demographic differences between the $\mathrm{OAB}$ and control groups and normalized for urine hydration using $\mathrm{Cr}$ levels. It also used a credible solidphase ELISA immunoassay modality geared strictly towards MCP-1. When seen in conjunction with results that have statistical significance concerning MCP-1 and symptom severity, these strengths provide substantial grounds to expect reproducibility, an area in which biomarker studies often struggle. Although in general MCP-1 cannot be used to assess the severity of diseases quantitatively, we found it to be closely linked to $\mathrm{OAB}$ and its clinical course.

With an AUC indicating that MCP-1 may have a promising diagnostic ability for $\mathrm{OAB}$, the $\mathrm{ROC}$ analysis suggests the potential use of MCP-1 immunoassays as a clinical tool to aid in the diagnosis of $\mathrm{OAB}$ and as a gauge for the effectiveness of a given treatment plan. The analysis of MCP-1 also brings to light opportunities for determining the severity of bladder inflammation present within an $\mathrm{OAB}$ patient and the identification of individuals with high MCP-1 levels in susceptible populations before the development of severe OAB-related symptoms. Prompt detection will allow the amelioration of $\mathrm{OAB}$ development by administering treatment earlier in the inflammatory development process. Conceivably, urinary MCP-1 levels and extensive $\mathrm{OAB}$ questionnaires, alone or in combination with adenosine triphosphate and interleukin-5 testing [25] can lead to novel noninvasive diagnostic tests for different $\mathrm{OAB}$ inflammatory phenotypes and may promote a more personalized management approach.

Nonetheless, the patient cohorts in the present study were still too small to establish definitive MCP-1 diagnostic guidelines for $\mathrm{OAB}$. The control, non-white, male, and neuromodulation subgroups were rather small and deserve more attention in future biomarker studies. Although the age difference between the $\mathrm{OAB}$ and control groups was insignificant $(\mathrm{P}=$ 0.078 ), the fact that the control patients were on average 8 years younger is worth noting because the frequency of chronic inflammatory diseases increases with age. This study did not include a voiding diary, although $\mathrm{OAB}$ is diagnosed mainly by urgency, which is better represented by symptom severity questionnaires such as $\mathrm{OAB}-\mathrm{V} 8$ and $\mathrm{PPBC}$ than a voiding diary. As of date, no MCP-1 urinalysis modality has been perfected in conformance with clinical standards. Despite these limitations, this study establishes the efficacy of urinary cytokine investigations for clinical usage, as well as for a greater understanding of 
the pathogenesis of $\mathrm{OAB}$.

In this analysis of urinary $\mathrm{MCP}-1$ levels in $\mathrm{OAB}$ patients, MCP-1 levels not only were higher in OAB patients, but also corresponded accurately to symptom severity scores and symptom responder/nonresponder classifications. Because of the complex mechanisms by which biomarkers operate, the variability between assay kits, and high or low endogenous biomarker levels, it may prove to be challenging to establish a single decisive urinary biomarker for OAB. Overall, our findings showed an excellent correlation of urinary MCP-1 levels with $\mathrm{OAB}$ symptoms and treatment response. These results suggest that MCP-1 may be a potential biomarker, alone or in multiplex with others, to aid in making a clinical diagnosis of $\mathrm{OAB}$, monitoring disease progression, and evaluating the response to treatment in $\mathrm{OAB}$ patients.

\section{ACKNOWLEDGEMENTS}

Authors would like to thank our patients and donors from UCI Health for contributing to our understanding of $\mathrm{OAB}$ disease.

\section{AUTHOR CONTRIBUTION STATEMENT}

- Conceptualization: $G G, B F, D C, F Z$

- Data curation: $B F, F Z$

- Formal analysis: $D C$

- Funding acquisition: $G G, D C, F Z$

- Methodology: $G G, B F, D C, F Z$

- Project administration: $G G$

- Visualization: $D C$

- Writing-original draft: $G G, B F, D C, F Z$

-Writing-review \& editing: $G G, B F, D C, F Z$

\section{REFERENCES}

1. Abrams P, Cardozo L, Fall M, Griffiths D, Rosier P, Ulmsten U, et al. The standardization of terminology of lower urinary tract function: report from the Standardization Sub-committee of the International Continence Society. Am J Obstet Gynecol 2002;187:11626.

2. Antunes-Lopes T, Pinto R, Barros SC, Botelho F, Silva CM, Cruz $\mathrm{CD}$, et al. Urinary neurotrophic factors in healthy individuals and patients with overactive bladder. J Urol 2013;189:359-65.

3. Bhide AA, Cartwright R, Khullar V, Digesu GA. Biomarkers in overactive bladder. Int Urogynecol J 2013;24:1065-72.
4. Kim JC, Park EY, Seo SI, Park YH, Hwang TK. Nerve growth factor and prostaglandins in the urine of female patients with overactive bladder. J urol 2006;175:1773-6.

5. Liu HT, Jiang YH, Kuo HC. Increased serum adipokines implicate chronic inflammation in the pathogenesis of overactive bladder syndrome refractory to antimuscarinic therapy. PloS One 2013;8:e76706.

6. Wang LW, Han XM, Chen CH, Ma Y, Hai B. Urinary brain-derived neurotrophic factor: a potential biomarker for objective diagnosis of overactive bladder. Int Urol Nephrol 2014;46:341-7.

7. Tyagi P, Barclay D, Zamora R, Yoshimura N, Peters K, Vodovotz Y, et al. Urine cytokines suggest an inflammatory response in the overactive bladder: a pilot study. Int Urol Nephrol 2010;42:629-35.

8. Ghoniem G, Faruqui N, Elmissiry M, Mahdy A, Abdelwahab H, Oomen $\mathrm{M}$, et al. Differential profile analysis of urinary cytokines in patients with overactive bladder. Int Urogynecol J 2011;22:953-61.

9. Deshmane SL, Kremlev S, Amini S, Sawaya BE. Monocyte chemoattractant protein-1 (MCP-1): an overview. J Interferon Cytokine Res 2009;29:313-26.

10. Naruse K, Ueno M, Satoh T, Nomiyama H, Tei H, Takeda M, et al. A YAC contig of the human CC chemokine genes clustered on chromosome 17q11.2. Genomics 1996;34:236-40.

11. Chocran BH, Reffel AC, Stiles CD. Molecular cloning of gene sequences regulated by platelet-derived growth factor. Cell 1983;33: 939-47.

12. Feuser K, Thon KP, Bischoff SC, Lorentz A. Human intestinal mast cells are a potent source of multiple chemokines. Cytokine 2012;58: 178-85.

13. Beall CJ, Mahajan S, Kuhn D, Kolattukudy PE. Site-directed mutagenesis of monocyte chemoattractant protein-1 identifies two regions of the polypeptide essential for biological activity. Biochem J 1996;313:633-40.

14. Jiang Y, Valente AJ, Williamson MJ, Zhang L, Graves DT. Posttranslational modification of a monocyte-specific chemoattractant synthesized by glioma, osteosarcoma, and vascular smooth muscle cells. J Biol Chem 1990;265:18318-21.

15. Desireddi NV, Campbell PL, Stern JA, Sobkoviak R, Chuai S, Shahrara S, et al. Monocyte chemoattractant protein-1 and macrophage inflammatory protein- $1 \alpha$ as possible biomarkers for the chronic pelvic pain syndrome. J Urol 2008;179:1857-62.

16. Tanaka ST, Martinez-Ferrer M, Makari JH, Wills ML, Thomas JC, Adams MC, et al. Recruitment of bone marrow derived cells to the bladder after bladder outlet obstruction. J Urol 2009;182:1769-74.

17. Farhan B, Chang H, Ahmed A, Zaldivar F, Ghoniem G. Characterizations of urinary monocyte chemoattractant protein 1: Potential biomarker for patients with overactive bladder. Arab J Urol 2019; 
17:58-60.

18. Lightner DJ, Gomelsky A, Souter L, Vasavada SP. Diagnosis and treatment of overactive bladder (non-neurogenic) in adults: AUA/ SUFU guideline amendment 2019. J Urol 2019;202:558-63.

19. Geppetti P, Nassini R, Materazzi S, Benemei S. The concept of neurogenic inflammation. BJU Int 2008;101:2-6.

20. Ziraldo C, Vodovotz Y, Namas RA, Almahmoud K, Tapias V, Mi Q, et al. Central role for MCP-1/CCL2 in injury-induced inflammation revealed by in vitro, in silico, and clinical studies. PLoS One 2013;8: e79804.

21. Yoshimura N, de Groat WC. Increased excitability of afferent neurons innervating rat urinary bladder after chronic bladder inflammation. J Neurosci 1999;19:4644-53.

22. Stephan M, Conrad S, Eggert T, Heuer R, Fernandez S, Huland H.
Urinary concentration and tissue messenger RNA expression of monocyte chemoattractant protein-1 as an indicator of the degree of hydronephrotic atrophy in partial ureteral obstruction. J Urol 2002;167:1497-502.

23. Firat E, Aybek Z, Akgun S, Kucuker K, Akca H, Aybek H. Exploring biomarkers in the overactive bladder: alterations in miRNA levels of a panel of genes in patients with OAB. Neurourol Urodyn 2019;38:1571-8.

24. Siddiqui NY, Hefand BT, Andreev VP, Kowalski JT, Bradley MS, Lai $\mathrm{HH}$, et al. Biomarkers implicated in lower urinary tract symptoms. J Urol 2019;202:880-9.

25. Firouzmand S, Ajori L, Young J. New participant stratification and combination of urinary biomarkers and confounders could improve diagnostic accuracy for overactive bladder. Sci Rep 2020;10:3085. 